\title{
Compression of Medical Images by Prediction on Wavelet Transform Coefficients
}

\author{
P.S. Arya Devi and M.G. Mini
}

\begin{abstract}
Compression of medical image is a challenging task as the compression has to be achieved without losing diagnostic quality of the images. Reducing the size of image finds its application in saving storage space and increasing transmission speed, as in case of teleradiology. On the decomposed details of an image, prediction is done. The high SNR (Signal to Noise ratio) and localization provided by wavelet coefficients makes it suitable for images. With few prediction coefficients, as small as 15, the whole image is reconstructed. The four variants of the method are studied to find its suitability for different types of images. The method is evaluated using various objective fidelity criteria.
\end{abstract}

Keywords--- HVS, Image Compression, Linear Prediction, Structural Similarity (SSIM), Teleradiology

\section{INTRODUCTION}

$\mathrm{C}$ COMPRESSION of medical images means expressing the image with less data to save storage space and transmission time, based on the prerequisite that true information in original image will be preserved. These images assist the radiologist in making high quality diagnosis and can be stored to facilitate archiving. Teleradiology is electronic transmission of radiological images like Computed Radiographic(CR), Computed Tomographic(CT), Magnetic Resonance(MR) and Ultrasound(US) for the purpose of interpretation and consultation. Teleradiology can provide image sharing, by which a centralized database for medical images for quick reference to obtain a correct diagnostic is possible. Teleradiology could also provide immediate consultation with remotely located specialists, which is beneficial to the patient. The basic services supported by an integrated teleradiology services network are: telediagnosis, teleconsultation, telemonitoring, and telemanagement[1].

Image compression is an important issue to be addressed in the area of transmission and storage of images. The storage and transmission of large volumes of image data is a challenging task owing to limited storage space and bandwidth. Even as the new emerging technologies promise unlimited bandwidth, the need and availability for images outgrow the

P.S. Arya Devi, Research Scholar, Department of Electronics, Model Engineering College, Kochi, India.E-mail:aryaps@mec.ac.in

M.G. Mini, Associate Professor, Department of Electronics, Model Engineering College, Kochi, India.E-mail:mininair@mec.ac.in

This work is supported by Engineering and Technology Programme, Kerala State Council for Science Technology and Environment.

DOI: 10.9756/BIJAIP.3085 increase in network capacity. The high costs involved in providing large bandwidth and huge storage space further necessitates the requirement for compression. Even as many techniques are available and emerging in the field of image compression, the demand for digital image transmission points to the fact that there is always room for better and new methods [2].

Discrete Wavelet Transform (DWT) decomposition provides a one-to-one representation of the signal in terms of its wavelet coefficients which are obtained as a result of reversible linear transformation. This makes DWT suitable for data compression [3]. DWT performs multi resolution analysis of an image to extract relevant information from an image and it can adapt to human visual characteristics. The image is decomposed into a set of different resolution sub-images corresponding to the various frequency bands and gives a multi-resolution representation of the image with localization in both spatial and frequency domains.

Images can be represented by homomorphic models in which linear prediction is compatible. Modelling a signal or system removes redundancy and this is the essence of compression [4],[5].

The image is decomposed using two-dimensional DWT (2D-DWT) and linear prediction is done on horizontal, vertical and diagonal detail coefficients and only small number of predictor coefficients and detail coefficients are retained in the compression. From the retained coefficients prediction is done to reconstruct the original image. Here we propose four methods for compression utilising prediction on horizontal, vertical and diagonal detail coefficients at each level.

\section{RELATED WORK AND BACK GROUND}

A brief revision of theory associated with the proposed method is given below. The areas dealt here are image compression fundamentals, wavelet transform, linear prediction and correlation properties of wavelet.

\section{A. Image Compression}

In most of the images, the neighboring pixels are correlated, and image contains redundant information. By compressing an image we should find a less correlated representation of the image. Image compression relies on reduction of redundancy and irrelevancy. Redundancy reduction removes duplication from image, and irrelevancy reduction omits parts of the signal that will not be noticed by Human Visual System (HVS). The redundancies in an image can be identified as spatial redundancy, coding redundancy and psycho visual redundancy. 
Image compression can be broadly divided into two categories: lossless and lossy compression. Lossless compression, also known as reversible compression guarantees the image after decompression to be identical to the original image. In case of irreversible compression, some information is removed permanently for reduction in size. In both the types, diagnostic quality of the image cannot be sacrificed. There are two ways by which we can assess compressed imagediagnostic accuracy and image quality perception. According to the recommendations by Royal College of Radiologists the acceptable compression ratio for CR and US images is 10:1 whereas for CT and MR it is 5:1 [6].

\section{B. Wavelet Transform}

Discrete dyadic wavelet transforms are invertible and efficient and have good spatial localization and fairly good frequency localization of their bases [7],[8]. When applied to an image, the image is split into its details at different scales, orientations and positions. The transformed image is decorrelated [9].

One-dimensional wavelet theory defines a function $\psi$, the wavelet, and it's associated scaling function $\varphi$, such that the family of functions $\left\{\psi^{j}(x)\right\} \mathrm{j} \in Z$, are orthonormal, where

$$
\psi^{j}(x)=\sqrt{2^{j}} \psi\left(2^{j} x\right)
$$

wavelet transform can be implemented by quadrature mirror filters $G=(g(n))$ and $H=(h(n)), n \in Z$, where

$$
g(n)=(-1)^{n} h(n)
$$$$
h(n)=1 / 2\langle\phi(x / 2), \phi(x-n)\rangle \text {; }
$$

( $<>$ denotes $L^{2}$ inner product). $H$ corresponds to a low-pass filter, and $G$ is an octave wide high-pass filter. The reconstruction filters have impulse responses [10]

$$
g^{*}(n)=g(l-n)
$$

$$
h^{*}(n)=h(l-n)
$$

A group of transform coefficients resulting from the same sequence of low pass and high pass filtering operations gives approximation and detail coefficients respectively.

Two dimensional wavelet transform is performed by applying a separable filter bank to the image. Applying the one dimensional transform in each row, we get two approximation and details coefficients in each row ( $L$ and $H$ subbands). Then applying a one dimensional DWT columnwise on these $L$ and $H$ subbands, four subbands $L L, L H, H L$, and $H H$ are obtained. $L L$ is a coarser version of the original input signal called approximation image. $L H, H L$, and $H H$ are the high frequency subbands containing the detail information (vertical, horizontal and diagonal details images). Figure 1 shows 2D DWT performed in separable form on an image and the two level decomposition of the image [2].

\section{Linear Prediction on Wavelet Coefficients}

Linear prediction model optimally extract information about a current sample from a neighborhood of samples in its causal past [4]. An image is a two dimensional signal, so it can be modeled as a signal. The signal is modeled as a linear combination of its past values and a hypothetical input to a causal system whose output is the given signal. The model parameters are obtained by a least squares analysis in the time domain and frequency domain [5]. The sample of the signal is predicted and if the prediction is done from weighted sum of other samples of the signal, the linear predictive model is auto regressive (AR) model [4]. The AR model of a process expresses it as finite linear aggregate of its previous values. Let us denote the values of stochastic process at equally spaced times, $n,(n-1),(n-2) \ldots$ by $y(n), y(n-1), y(n-2), \ldots \ldots$

$y(n)=\alpha_{1} y(n-1)+\alpha_{2} y(n-2)+\cdots \ldots+\alpha_{p} y(n-p)$

The Yule-Walker equations are obtained by exploiting autocorrelation values and these equations are solved to get predictor values $\alpha$.

Prediction on wavelet coefficients exploits the correlational properties of wavelet transform coefficients. Let $C A_{j}$ and $C D_{j}$ be the approximation and details coefficients of a signal at decomposition level $j$. The expectation of approximation coefficients is proportional to the average of the original signal. It can also be found that detail coefficients have zero mean.The autocorrelation of $C A_{j}$ and $C D_{j}$ are proportional to autocorrelation of original signal. $C A j$ and $C D j$ are decorrelated. The property of localized WT coefficients will lead to more correlation of wavelet coefficients in the same prediction channel. The higher the correlation, the more is the scope for redundancy removal in prediction filtering [11]. 


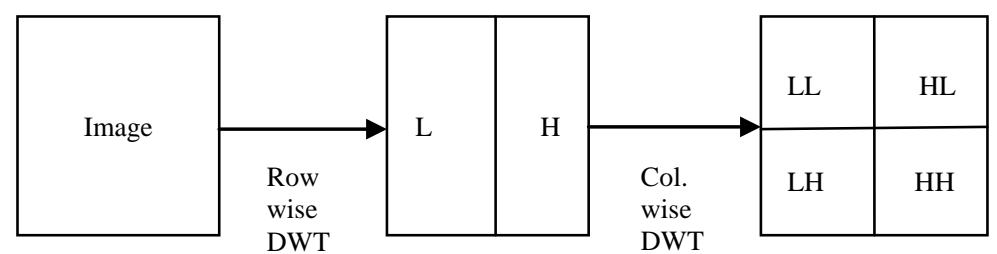

(a)

\begin{tabular}{|l|l|l|}
\hline LL2 & HL2 & \multirow{2}{*}{ HL1 } \\
LH2 & HH2 & \\
\hline LH1 & HH1 \\
& & \\
\hline
\end{tabular}

(b)

Figure 1: 2D-DWT a) First level decomposition b) Second level decomposition

\section{THE PROPOSED METHOD}

In the proposed method 2D-DWT is performed on the original image up to four levels. The image is decomposed to approximation and detail subbands. All the coefficients of fourth level subbands are retained. Prediction is done for all detail coefficients from level three to level one. A small number of prediction coefficients and that much detail coefficients, at each level along with retained coefficients from fourth level forms compressed image.

In the decompression, with small number of retained predictor coefficients and that much detail coefficients in the $i^{\text {th }}$ level, all the details coefficients in the $i^{\text {th }}$ level are reconstructed. Approximation coefficients for $i^{\text {th }}$ level is obtained from inverse DWT on $(i+1)^{\text {th }}$ level of wavelet coefficients. Using approximation and detail coefficients of $i^{\text {th }}$ level approximation of $(i-1)^{\text {th }}$ level is obtained. Thus the whole image is reconstructed. We have four variants of the proposed algorithm. In the first variant row wise prediction of subband coefficients is done whereas the second one predicts columnwise. In the third and fourth variants, horizontal subbands coefficients are predicted row-wise and vertical subbands coefficients are predicted column-wise. The two differs in the handling of diagonal coefficients. The wavelet used here is bior $9 / 7$.

\section{A. Row/Column-wise Prediction for all Subbands}

The algorithm for the first two variants is given as follows.

\section{Algorithm}

\section{Compression}

1. Step1: Select the image of interest and input the no. of coefficients, $n$ to be retained.

2. Step2: Perform DWT on the image.

Decompose the image into wavelet transform coefficients applying 2D- DWT on the image.
3. Step3: Retaining the coefficients

Retain the fourth level decomposition coefficients so that they can be used for reconstruction. In addition to that $n$ rows/columns of each level of all horizontal, vertical and diagonal subbands are also retained.

4. Step4: Obtain the predictor coefficients.

Take the first row/column and find $n$ predictors for first row/column of detail coefficients. Similarly find $n$ predictors for all rows/columns. Do this for all the subband levels and orientations

Decompression

Step1: Reconstruction of detail coefficients.

a) Initialise $i=4$.

b) Initialise an array for predicted coefficients.

c) Using first $n$ coefficients in the row/column and $n$ predictors the whole row/column is reconstructed. It is done for all of rows/columns.

Step2: Reconstruction of approximation coefficients

Applying 2D-IDWT on the $(i+1)^{\text {th }}$ level coefficients, $i^{\text {th }}$ level approximation is reconstructed.

Step3: Reconstruction of previous levels.

Steps 1 and 2 are repeated till $i=$ zero. Thus the original image is reconstructed from prediction

\section{B. Column/Row-wise prediction for Diagonal Subbands}

It was found from [12] that for images with horizontal orientation row-wise prediction is beneficial whereas for images with vertical orientation column-wise prediction is better. Based on this the following algorithm was developed.

\section{Algorithm}

\section{Compression}

Step1: Select the image of interest and input the no. of coefficients, $n$ to be retained.

Step2: Perform DWT on the image. 
Decompose the image into wavelet transform coefficients applying 2D- DWT on the image.

Step3: Retaining the coefficients

Retain the fourth level decomposition coefficients so that they can be used for reconstruction. In addition to that $n$ rows/columns of each level of all horizontal, vertical and diagonal subbands are also retained.

Step4: Obtain the predictor coefficients.

a) Take the horizontal subband first. Take first row and find $\mathrm{n}$ predictors for first row of horizontal coefficients. Similarly find $\mathrm{n}$ predictors for all rows.

b) Next take vertical subband. Take first column and find $\mathrm{n}$ predictors for first column of vertical coefficients. Similarly find $\mathrm{n}$ predictors for all columns.

c) For diagonal subband, obtain the diagonals. Arrange them such that each diagonal form a row. Pad with zeros wherever necessary. Take first column/row in the resultant matrix and find $\mathrm{n}$ predictors for first column/row. Similarly find $\mathrm{n}$ predictors for all columns/rows.

d) Do this for all the subband levels.

\section{Decompression}

Step 1: Reconstruction of detail coefficients.

a) Initialise $i=4$.

b) Initialise an array for predicted coefficients. Using first $n$ coefficients in the row and $n$ predictors the whole row is reconstructed. It is done for all of rows. Thus horizontal subband is reconstructed

c) Initialise an array for predicted coefficients. Using first $n$ coefficients in the column and $n$ predictors the whole column is reconstructed. It is done for all of columns. Thus vertical subband is reconstructed

d) Step c/b is repeated for rearranged diagonal sub- band. The excess zeros are removed and reconstructed diagonal subband. The excess zeros are removed and reconstructed diagonal subband is rearranged to look similar to original diagonal subband.

Step2: Reconstruction of approximation coefficients

Applying 2D-IDWT on the $(i+1)^{\text {th }}$ level coefficients, $i^{\text {th }}$ level approximation is reconstructed.

Step3: Reconstruction of previous levels.

Steps 1 and 2 are repeated till $i=$ zero. Thus the original image is reconstructed from prediction.

\section{EVALUATION CRITERIA}

The reconstructed image can be assessed by two classes of criteria; objective fidelity criteria and subjective fidelity criteria. In this paper we deal with some of the objective fidelity criteria. In objective fidelity criteria the level of information loss is expressed as a function of input image, compressed image and subsequently decompressed image [13]. Normalised mean square error (NMSE), Normalised Absolute Error (NAE) and peak signal to nose ratio (PSNR) are among the objective fidelity criteria [14]. Even though MSE or PSNR are easy to calculate and deal with for optimizations, they may not correlate well with the human perception of quality. So we go for structural similarity (SSIM). The task of similarity measurement is separated into three comparisons: luminance, contrast and structure.
Structural similarity (SSIM) index between signals $\mathrm{x}$ and $\mathrm{y}$ is given as

$$
\left.\operatorname{SSIM}(x, y)=[l(x, y)]^{\alpha} \cdot[c(x, y)]\right]^{\beta} \cdot[s(x, y)]^{\gamma}
$$

where $\alpha=\beta=\gamma=1$.SSIM values range from 0 to 1 [15].

Let $x(m, n)$ be the original $\mathrm{M} \times \mathrm{N}$ pixel image and $x^{*}(m, n)$ be the reconstructed image

$$
\begin{aligned}
N M S E & =\frac{\sum \sum\left[x(m, n)-x^{*}(m, n)\right]^{2}}{\sum \sum[x(m, n)]^{2}} \\
P S N R & =10 \log _{10} 2^{b}-1 / M S E
\end{aligned}
$$

Mean SSIM is used to evaluate overall image quality. It is obtained as

$$
\operatorname{MSSIM}(X, Y)=\frac{1}{M} \sum_{j=1}^{M} \operatorname{SSIM}\left(x_{j}, y_{j}\right)
$$

where $\mathrm{X}$ and $\mathrm{Y}$ are the reference and the distorted images, respectively; $\mathrm{x}_{j}$ and $\mathrm{y}_{j}$ are the image contents at the $j^{\text {th }}$ local window; and $M$ is the number of local windows in the image.

\section{RESULTS}

The dataset used here is the medical image set made available by Roman Starosolski. The set contains medical images of various modalities (CR, CT, MR, and US) stored in the PGM P5 format [16]. The biorthogonal wavelet which is suited for image compression is used here. CR images considered here are of dimensions greater than $1024 \times 1024$. $\mathrm{CT}$ and MR images have $512 \times 512$ dimensions. US images are of the size $640 \mathrm{x} 480$.The performance measures are plotted as shown in Figure 2. 

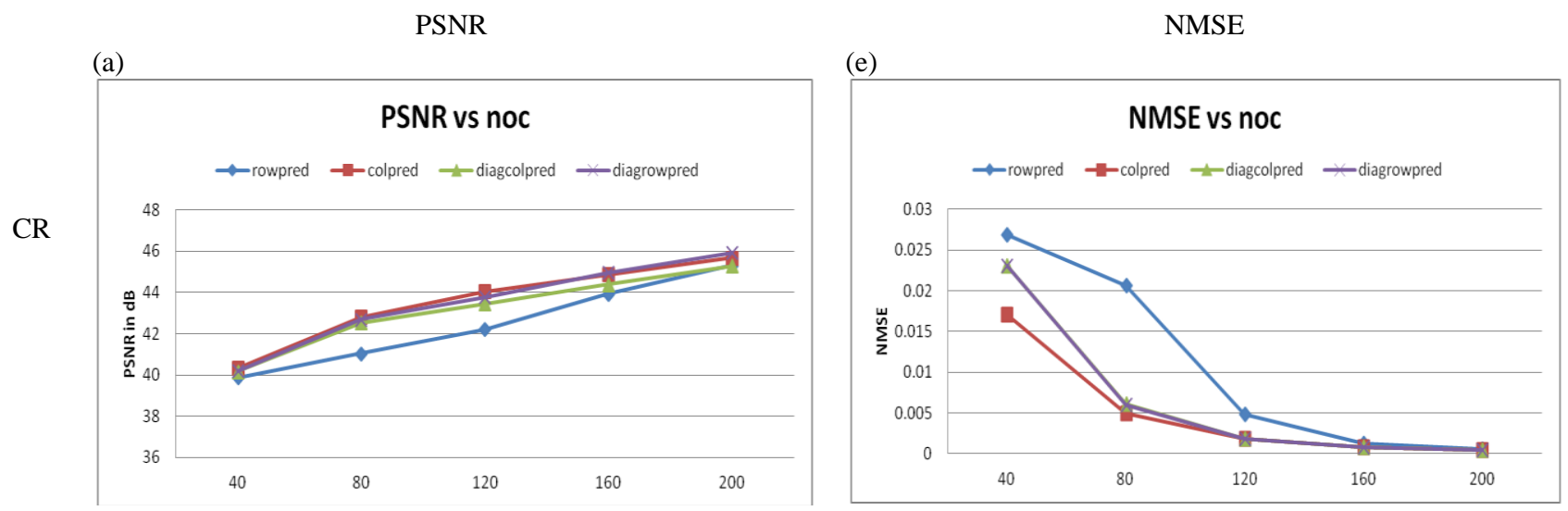

(b)
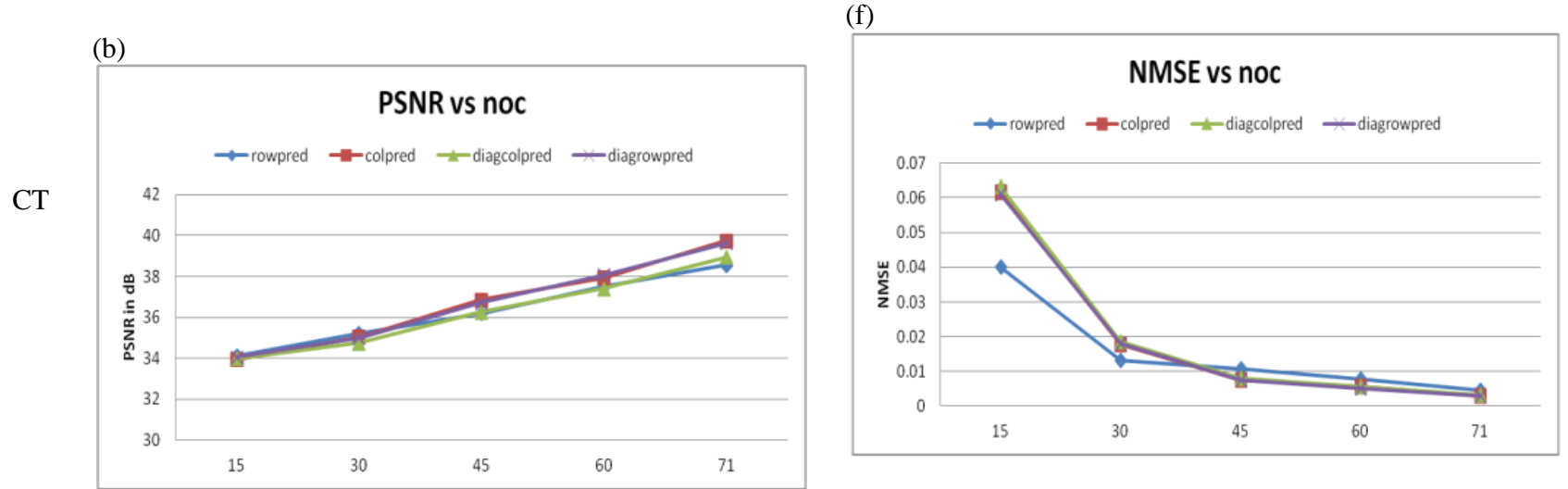

(c)

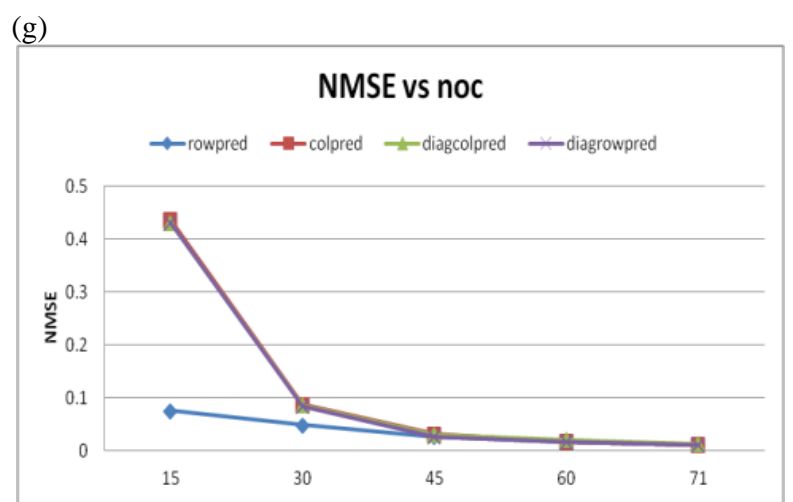

(d)

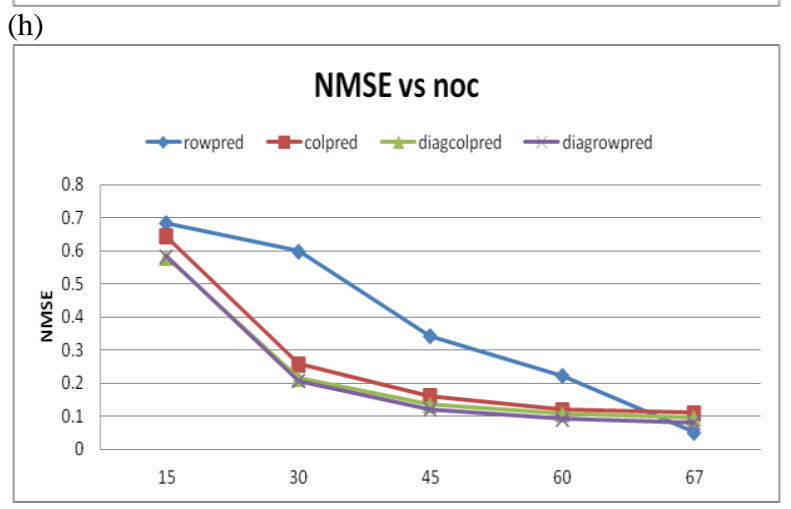

NAE

MMSIM 
(i)

CR

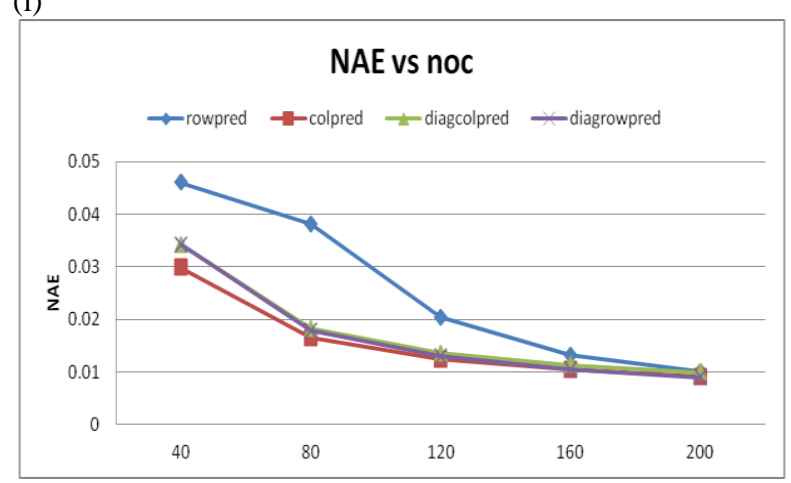

(j)

CT

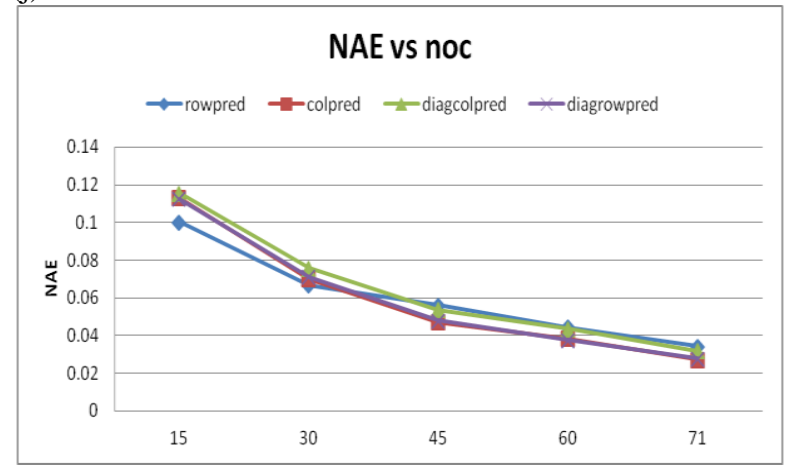

(k)

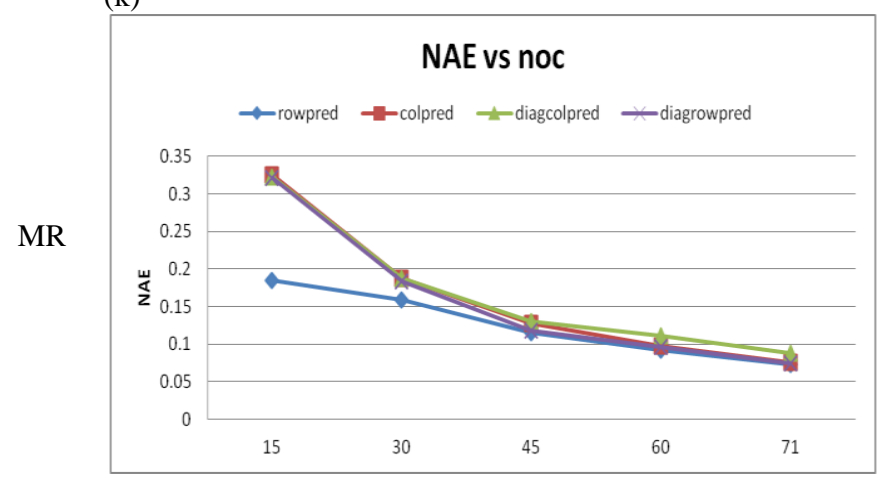

(1)

US

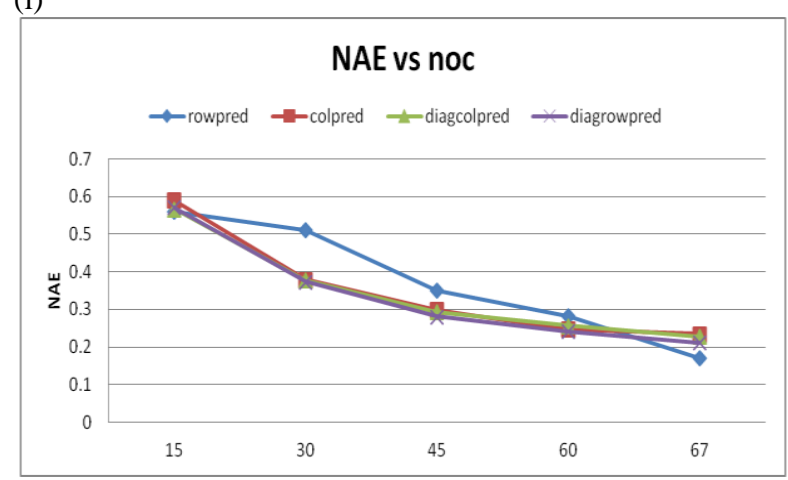

(m)

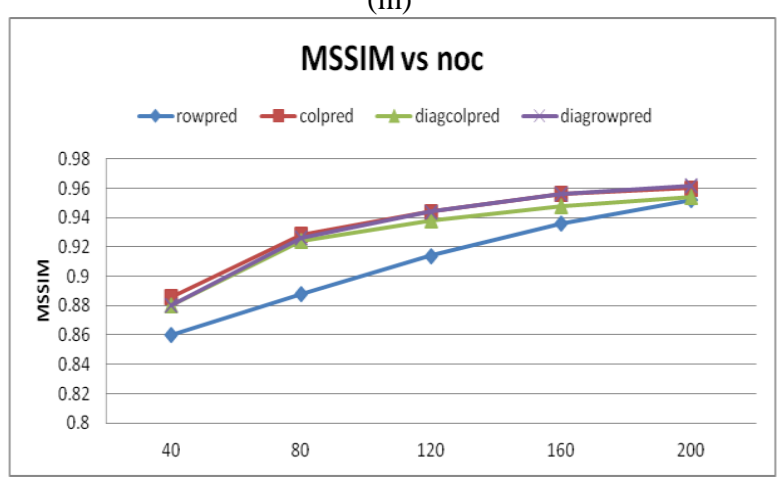

(n)

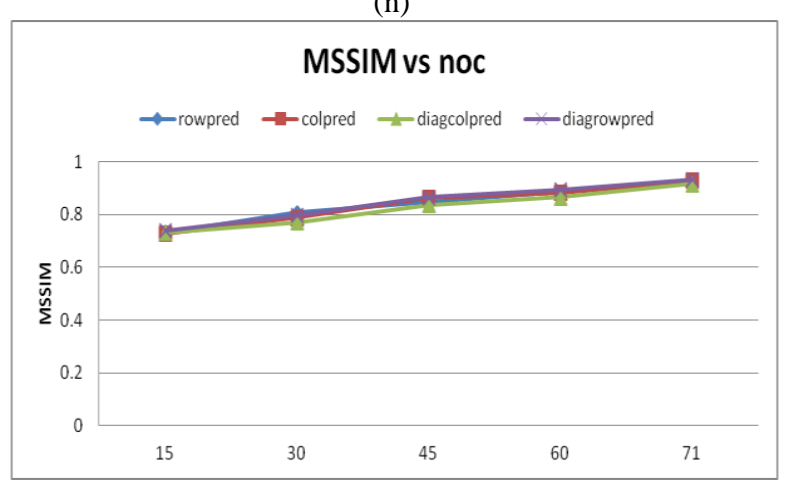

(o)

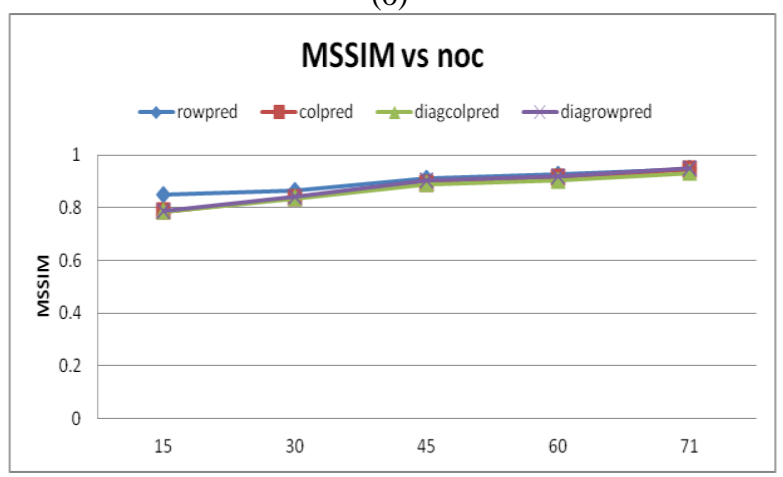

(p)

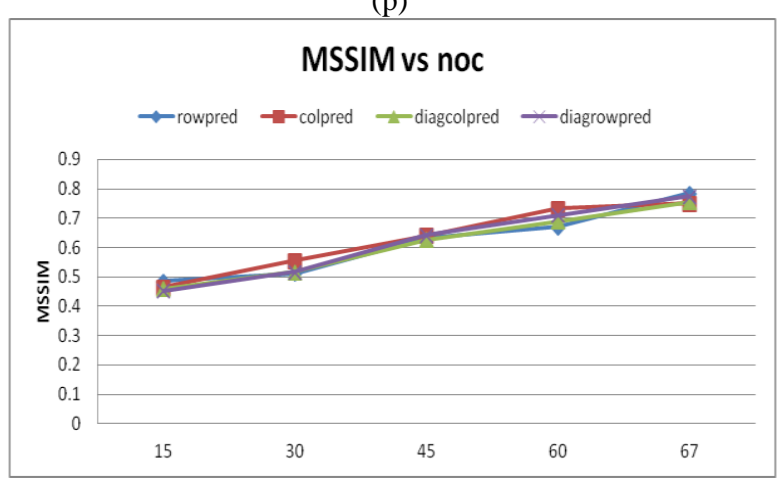

Figure 2: Performance Measures vs. no. of Coefficients Retained

(a-d): PSNR, (e-h): NMSE, (i-l): NAE, (m-p): MSSIM 
The performance of the algorithm is validated using objective criteria like PSNR, NMSE, NAE and MSSIM. It is found that as the number of coefficients retained increases PSNR and MSSIM increase whereas NMSE and NAE decrease. For CR images column-wise prediction for diagonal subband method is best suited. PSNR for CR image goes as high as $45.92 \mathrm{~dB}$ for 200 retained coefficients in this method. NMSE and NAE gives values 0.000393 and 0.0090354 respectively whereas MSSIM has the value 0.962 for 200 retained coefficients.

CT images have high MSSIM for column-wise prediction for diagonal subband method. Column-wise prediction for diagonal subband method and column-wise prediction for all subband method show similar performance in the case of PSNR. In case of MR images row-wise prediction for all bands shows slightly better performance than other methods when no. of retained coefficients is less. As no. of coefficients increases, the performance becomes at par with other methods. All these four algorithms are not suited for US images. MSSIM values range from 0.4 to 0.79 .

The original and reconstructed images are shown in Figure3. The CR image contains lesser amounts of details. The detail is reduced and limited by the blurring that occurs at different stages of the imaging process. The amount of blurring depends upon the size and location of the object, motion and light spreading within the fluorescent screen. When an image is divided into pixels, some amount of blurring occurs. The pixel size is also a factor that contributes to limiting of details. So the method described here is suitable for CR images [17].

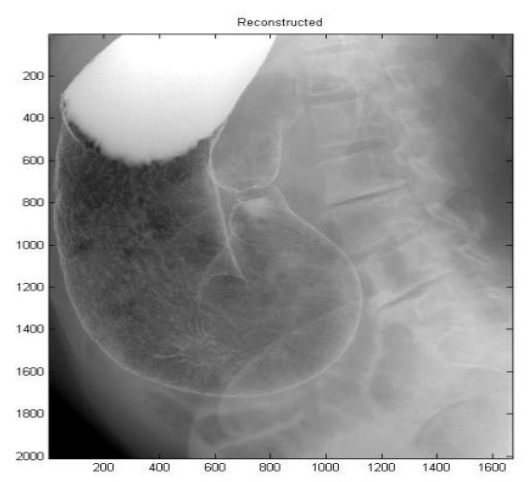

(a2)

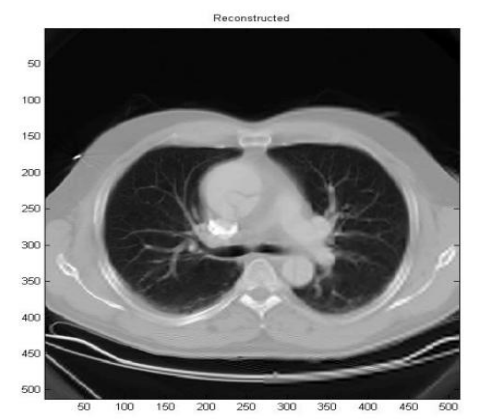

(b2)

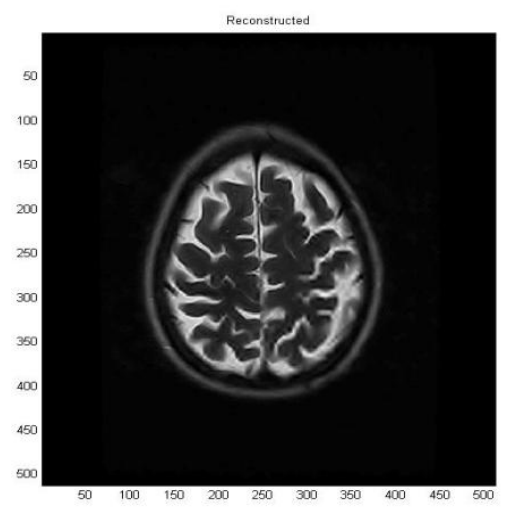

(c2) 
US

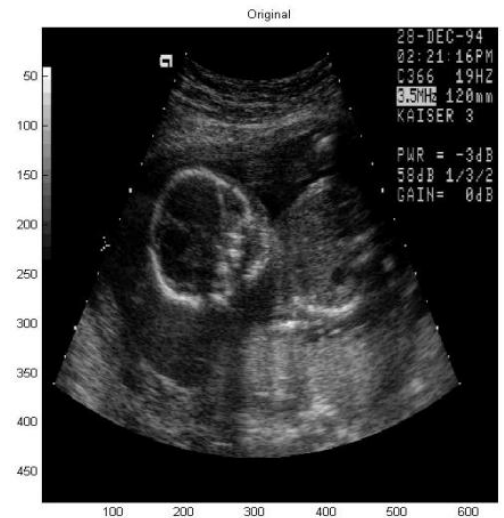

(d1)

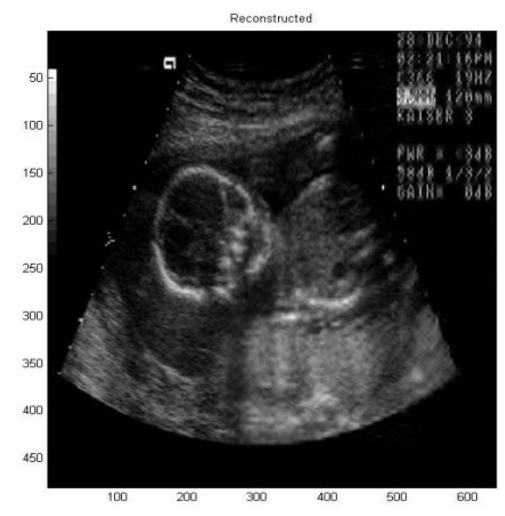

(d2)

Figure 3: (a1-d1) Original Images: (a2-d2) Reconstructed Images

\section{CONCLUSION}

In this method the correlation among the detail wavelet coefficients exploited to predict the next wavelet coefficients. Four variants of the proposed method are evaluated. The performance of method is evaluated on the basis of subjective criteria parameters like MSSIM and NMSE. The experimental results show that the proposed method, gives good compression and better quality images. We can conclude that for CR images the method works best and for US images it gives poor performance.

\section{REFERENCES}

[1] Stelios C. Orphanoudakis, Eleni Kaldoudi and Manolis Tsiknakis, "Technological Advances in Teleradiology", Eur. J. Radiology, Vol. 22, No.3,Pp. 205-217, 1996.

[2] P.S. Arya Devi and M.G. Mini, "Compression of Gray Scale Images Using Linear Prediction on Wavelet Coefficients ", Advances in Computer Science and Information Technology, Proc. CCSIT 2012, Vol. 86, No. 3, Pp. 49-58, 2012.

[3] M. Unser, "Wavelets, statistics, and biomedical application", 8th IEEE Signal Processing Workshop on Statistical Signal and Array Processing (SSAP), Pp.244-249, 1996.

[4] R. Rajagopalan, M.T Orchard, and K. Ramchandran, "Optimal Supports for Linear Predictive Models," IEEE Trans. Signal Processing, Vol. 44, No. 12, Pp. 3150-3153, 1996.

[5] J .Makhoul, " Linear Prediction: A tutorial review," Proc. IEEE, Vol 63, No. 4, Pp. 561-579, 1975

[6] The Royal College of Radiologists: The adoption of lossy image data compression for the purpose of clinical interpretation, 2008. Available at http://ww.rcr.ac.uk/docs/radiology/pdf/IT_guidance_LossyApr08.pdf

[7] G. Strang, and T. Nguyen, Wavelets and Filter Banks. Wellesley Cambridge Press, Wellesley M A, 1996.

[8] S. Mallat, A Wavelet Tour of Signal Processing. Academic Press, London, 1999.

[9] A. M. C. Ruedin and D G Acevedo, "Prediction of coefficients for Lossless Compression of Multispectral Images," Satellite Data Compression, Communications, and Archiving, SPIE, Vol. 5889, , Pp. 202-211, 2005.

[10] A. S Lewis and G Knowles, "Image Compression Using the 2-D Wavelet Transform,". IEEE Trans. Image Processing, Vol.1, No. 2, Pp. 244-250, 1992.

[11] J. S Huang, D.T Nguyen, M. Negnevitsky and C. J. E Philips, “ Correlation Properties of Wavelet Transform and Applications in Image Coding," Proc. IEEE Symp. Signal Processing and its Applications, Vol.2, Pp. 611-614, 1999.

[12] P S Arya Devi and M G Mini, " "Gray Scale Image Compression based On Wavelet Transform and Linear Prediction", The International Journal of Multimedia and its Applications (IJMA), Vol.4, No.1, Pp. 47-62, 2012.
[13] R.C Gonzalez, and R. E Woods, Digital Image Processing. PrenticeHall. India (2006).

[14] E. Shahhoseini, N. A Nejad, H. Behnam and A. Shahhoseini, " A new approach to compression of medical ultrasound images using wavelet transform," IEEE Conf. on Advances in Circuit, Electronics and Microelectronics, Pp. 40-44, 2012.

[15] Zhou Wang, Alan C. Bovik, Hamid R. Sheikh and Eero P. Simoncelli," Image Quality Assessment: From Error Visibility to Structural Similarity", IEEE Transactions On Image Processing, Vol. 13, No. 4, pp 600-612, 2004

[16] http://sun.iinf.polsl.gliwice.pl/ rstaros/mednat/index.html.

[17] P S Arya Devi and M G Mini, "Compression of Computed Radiographic Images using Linear Prediction onWavelet Coefficients", Advances in Computing and Communications, International Conference (ICACC), IEEE Xplore, Pp 130 - 133, 2012.

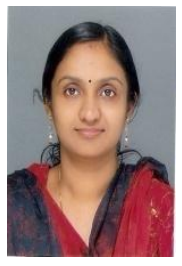

P.S. Arya Devi is Research Scholar at Model Engineering College, Kochi, Kerala. She obtained her B.E Degree Electronics \& Communication Engineering from Bharatidasan University, received M.Tech in Applied Electronics from Mahatma Gandhi University, Kerala in 2008. Her areas of interest include Image Compression, Information Theory and Coding. (E-mail: aryaps@mec.ac.in)

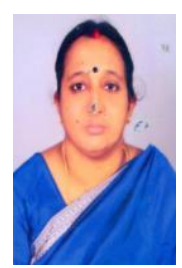

M. G. Mini is Associate Professor and Academic Head of Model Engineering College, Kochi, Kerala. She has received PhD from CUSAT, Kerala in 2005. Her areas of interest include Digital Image Processing, VLSI, etc. She has several research papers published in National and International journals to her credit. (E-mail: mininair@mec.ac.in) 